\title{
Analyzing the Resonance Resultant from the Capacitive Effects in Bulk Current Injection Probe
}

\author{
Issa Moustafa MASHRIKI, Seyyed Mohamad Javad RAZAVI, Seyyed Hossein MOHSENI ARMAKI
}

Faculty of Electrical \& Computer Engineering, Malek Ashter University of Technology, Iran

Issa_mashriki@yahoo.com, \{razavismj, mohseni\}@mut.ac.ir

Submitted November 25, 2019 / Accepted January 23, 2020

\begin{abstract}
In this paper, the effect of the slot distance, existed between the two parts of BCI-probe shield, on its performance is studied, therefore, two ferrite materials with the same size and different characteristics are used to implement two identical BCI-probes. The input impedance and reflection coefficient are measured for four different values of slot distance 0.5, 1, 2 and $3 \mathrm{~mm}$. Practical results show a notable effect of the slot distance on measured quantities.
\end{abstract}

Measured impedance analysis shows the appearance of three capacitive regions in its spectra, therefore, a three dimensional electromagnetic (EM) model is implemented in CST-Microwave Studio (MWS) software to disclose the main source parameters responsible for it.

Results of achieved study, using developed EM model, were in accordance with measured data for the implemented prototypes, and showed the same resonance phenomena.

\section{Keywords}

Bulk current injection (BCI) probe, electromagnetic compatibility (EMC), shield effect, slot capacitance, dielectric constant of ferrite

\section{Introduction}

Bulk current injection probe is a wide band low insertion loss power transformer, when clamped on a wire harness it produces in it an electromagnetic interference, it has the ability to identify the source of problem, therefore, the BCI probe is widely used in testing the susceptibility or immunity of electronic systems. Because of its advantages, and low cost compared to antenna procedures, BCI techniques were accepted by about all regulatory standards [1-6].

The shield is an essential part of any BCI probe; its main purpose is to prevent any possible electromagnetic interference with and from the device. It is provided, from its inner side, with a small gap or slot as shown in Fig. 1(a), thus, the probe is not fully shielded. This slot cannot be avoided, otherwise, the probe would be short circuited; and its distance must be small to decrease the said interference.

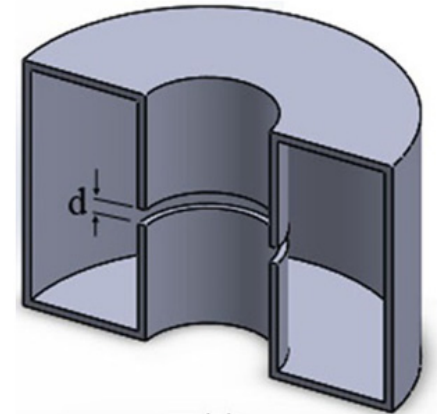

(a)

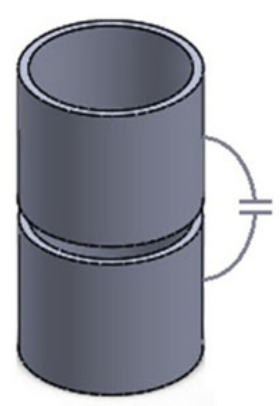

(b)
Fig. 1. (a) A cross section of BCI-probe shield, (b) the inner part of the shield.

The shield produces capacitive effects in the probe, which reduces its performance, there is no previous research to assess how the shield can affect the properties of the probe.

An accurate prediction of injection behavior is an important step, it helps in estimating and controlling RF interference levels, therefore, several research efforts have done for modeling, analyzing and characterizing the BCI probe in order to study a specific setup [7-10], or to improve its high frequency performance [11]. Further works use the circuit concept approach which replaces the probe with its equivalent source voltage and current without taking into account its main characteristics [12-14]. Other works have benefit from software tools for optimizing the model or achieving needed refinements in evaluating the circuit parameters [15], [16], or for implementing a $2 \mathrm{D}$ or $3 \mathrm{D}$ electromagnetic models [17-19], in which the complex permeability spectra is the main parameter that should be known.

Nevertheless, in spite of the good accordance of some developed models with measurements, a model that characterizes the BCI-probe apart from its internal structure is not available. For example, there is no circuital model which takes the slot, inter winding and ferrite dielectric constant capacitive effects into consideration.

For the primary turn, the shield represents a secondary coil loaded with the slot capacitance. The total value of this capacitance is usually small and can be neglected at low frequencies, therefore, it is treated as an open circuit. At high frequencies the impedance of this capacitance de- 
creases dramatically and its impact becomes meaningful, this is because of its parallel connection with the probe main inductance.

In this work, the role of the shield, connector and ferrite permittivity on the resonance phenomena observed in most BCI-probes is studied. This helps in understanding their effect on the probe's performance in the aim of improving it. In modeling, it helps in disclosing the role of each parameter and its location in the developed circuital model which leads to derive an accurate one. Firstly, the effect of the slot capacitance on measured reflection coefficient, and input impedance is shown practically by implementing two BCI-probes, then, a detail analysis and interpretation for the results is made using CST-MWS by implementing a 3D EM model.

\section{Resonance Phenomenon in BCI- Probe}

The input impedance spectra of the probe, in case of clamping it on a fixture or not, can be expressed in complex notation as $\hat{Z}(f)=R(f)+\mathrm{j} X(f)$, where $R(f)$ is the real part and $X(f)$ is the imaginary part of $\hat{Z}(f)$. At the frequency $f_{0}$ at which resonance occurs, $X\left(f_{0}\right)=0$, and $\hat{Z}\left(f_{0}\right)=R\left(f_{0}\right)$ coincide a peak of the curve. This form of resonance appearance was measured in [10] (Fig. 6 \& Fig. 7) and [15] (Fig. 3) for the BCI-probe model FCC-F140, and in [17] (Fig. 6 \& Fig. 9) for the probe model FCC-F120 8G in case of clamping and non-clamping it on a fixture, respectively.

From the definition of reflection coefficient spectra $\hat{S}_{11}=(\hat{Z}-50) /(\hat{Z}+50)$, when the imaginary part of $\hat{Z}$ is zero, the imaginary part of $\hat{S}_{11}$ is zero, too. Thus, the frequency at which resonance occurs is characterized by a magnitude of $\hat{S}_{11}$ equal to its real part, and small value of imaginary part in $\mathrm{dB}$. This form of resonance appearance was measured in [19] (Fig. 7) for the probe model FCCF130A, and [15] (Fig. 7) for FCC-F140, in case of nonclamping the probe on a fixture; and in [9] (Fig. 14) for FCC-F120-2 and [9] (Fig. 16) for FCC-F130-A, in case of clamping the probe on a fixture.

The working band of frequency of some BCI-probes, from Fischer Custom Communications (FCC) [20], and their measured resonance frequency in case of not clamping it on a fixture, are listed in Tab. 1. It shows that, for the proposed models, two resonance frequencies exist within the probe's working bandwidth, which proves that these BCI-probes include capacitive working modes. Compared to the probe's usable frequencies, the first resonance appears at a relatively low frequency, which imposes

\begin{tabular}{|c|c|c|c|c|}
\hline Model & $\begin{array}{c}\text { Usable freq. } \\
{[20]}\end{array}$ & $\begin{array}{c}\mathbf{1}^{\text {st }} \text { resonance } \\
{[\mathrm{MHz}]}\end{array}$ & $\begin{array}{c}\mathbf{2}^{\text {nd }} \text { resonance } \\
{[\mathrm{MHz}]}\end{array}$ & Ref. \\
\hline F130A & $1-450 \mathrm{MHz}$ & 35 & 300 & {$[19]$ (Fig. 7) } \\
\hline F140 & $1-1000 \mathrm{MHz}$ & 35 & 650 & {$[15]$ (Fig. 3) } \\
\hline F120-8G & $0.01-400 \mathrm{MHz}$ & 10 & 90 & {$[17]$ (Fig. 6) } \\
\hline
\end{tabular}

Tab. 1. BCI-probe models from FCC and their resonance frequencies. a question about the way at which the probe couples the wire harness above this limit.

\section{Injection Probe Characterization}

The BCI probe is a wide band magnetic device, with mostly one turn primary coil wound around a toroidal ferrite core. Figure 2 illustrates a radial cross sectional view of a typical BCI probe, the terminals of the primary coil are connected to N-type $50 \Omega \mathrm{RF}$-connector, one terminal is attached to the inner pin while the other one is connected to the external shield of the connector. The probe is shielded using two identical aluminum frames connected together with the outer shield of the connector from the outer side of the probe, and separated by a small slot from the inner one.

When the BCI-probe is not clamped on a wire under test, the shield is seen by the primary as a secondary coil, with one turn, loaded by the slot capacitance as shown in Fig. 3(a), where $C_{\mathrm{S}}$ and $C_{\mathrm{w}}$ denote the slot and inter winding capacitors, respectively. Thus, clamping the probe on a wire, adds another secondary coil, and both secondary coils must be taken into account in any derived circuital model. In case of small $C_{\mathrm{w}}$, the effect of just $C_{\mathrm{S}}$ remains, when transformed to the primary it can be represented as shown in Fig. 3(b). Its connection in parallel with the primary inductor, means that $C_{\mathrm{S}}$ could generate a resonance at a certain frequency, above which the input impedance becomes predominantly capacitive and the effectiveness of the ferrite core is degraded, especially for high value of $C_{\mathrm{S}}$.

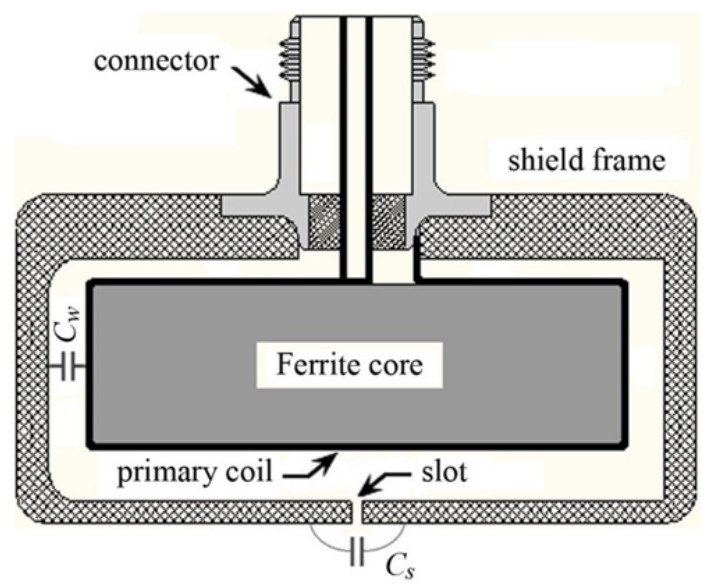

Fig. 2. Radial cross-section of a general type BCI probe with its input connector.

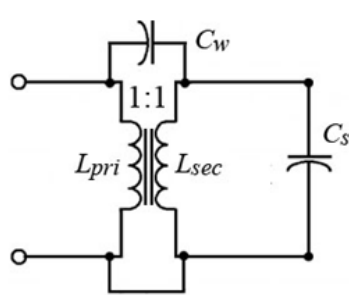

(a)

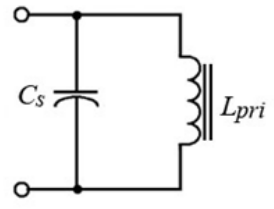

(b)
Fig. 3. (a) The connection of the shield capacitors and (b) the equivalent model showing the effect of just the slot capacitance after transforming the secondary to primary. 


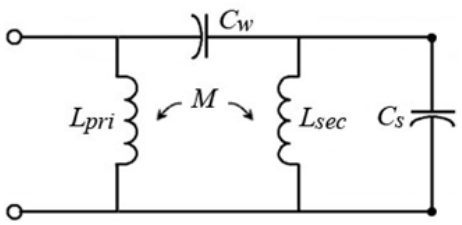

Fig. 4. The equivalent model for the probe showing the effect of comparable inter winding capacitance effect.

In case of comparable inter winding capacitance, the transformer can be modeled with two coupled inductors $L_{\text {pri }}$ and $L_{\text {sec }}$, with mutual inductance $M$ as shown in Fig. 4 . The capacitive effect resultant from the connector adds another, in parallel, capacitor at the input of the model.

\section{Verifying the Slot Capacitive Effect}

To evaluate the influence of the slot capacitance on measured reflection coefficient parameter, four toroidal cores from Fair-Rite company model 5961003801 (\#61 NiZn material), with the permeability spectra shown in Fig. 5 [21], are used in fabricating the BCI-probe shown in Fig. 6, the design takes into regard to use the same primary winding and to achieve the same spaces between the core and the shield as that for F-130A from FCC [20].

To achieve the needed tests using vector network analyzer (VNA) model HP8510, the device is provided by SMA-to-N type adapter to allow the use of coaxial cables equipped with SMA connectors. The available minimum testing frequency of proposed VNA is $45 \mathrm{MHz}$, a device with lower range is not at the authors' disposal, but since the effect of the slot capacitance appears at high frequencies, it would be sufficient. The VNA is calibrated in the frequency range $45-1100 \mathrm{MHz}$, and measurements are carried out at this frequency interval.

The amplitude of reflection coefficient is measured for four different slot spacing distances $(0.5,1,2$ and $3 \mathrm{~mm})$. The results of measurement are plotted together in Fig. 7, it shows that the slot distance has a notable effect on measured reflection coefficient. When the slot distance increases, the value of its associated capacitor decreases, which increases its impedance, and thus, the frequency at which the shield behaves as a short circuit, this explains the minimum shift towards higher frequencies by increasing the slot distance.

The precedent procedure is repeated to the core model 710-74270097 (material 4W620) from Wurth Electronics which has the same dimensions as the previous one, but with higher initial permeability of 620 instead of 125 for Fair-Rite core material. The results of measurements are shown in Fig. 8, the same resultant minimum shift is observed. This proves that the effect of slot capacitance can't be neglected while designing and modeling the BCI-probe. The common point between Figs. 7 and 8 is that, even though two materials with different characteristics are used, minimums of $S_{11}$ occur at the same frequencies, which means that these minimums don't relate to the core material or permeability.

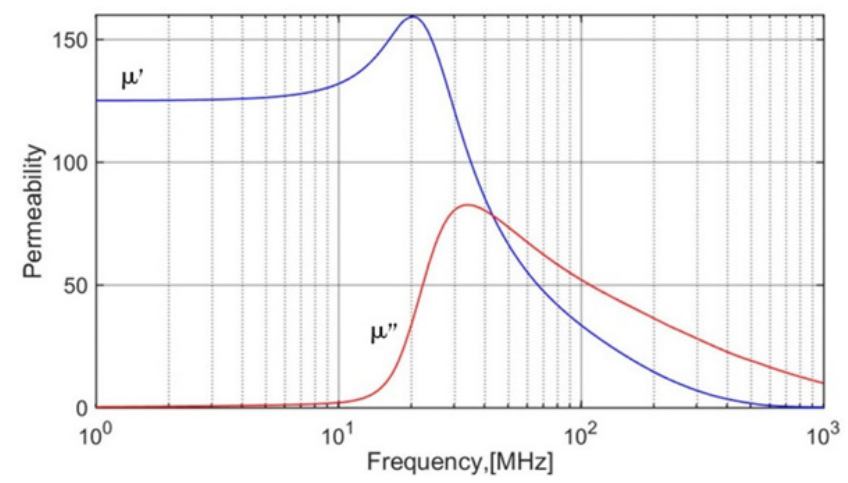

Fig. 5. Complex permeability spectra for material \#61 from its catalog [21], real part (blue) and imaginary part (red).

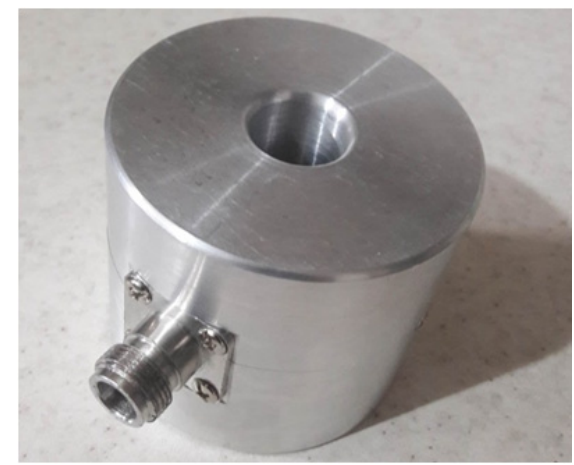

Fig. 6. Fabricated BCI-probe.

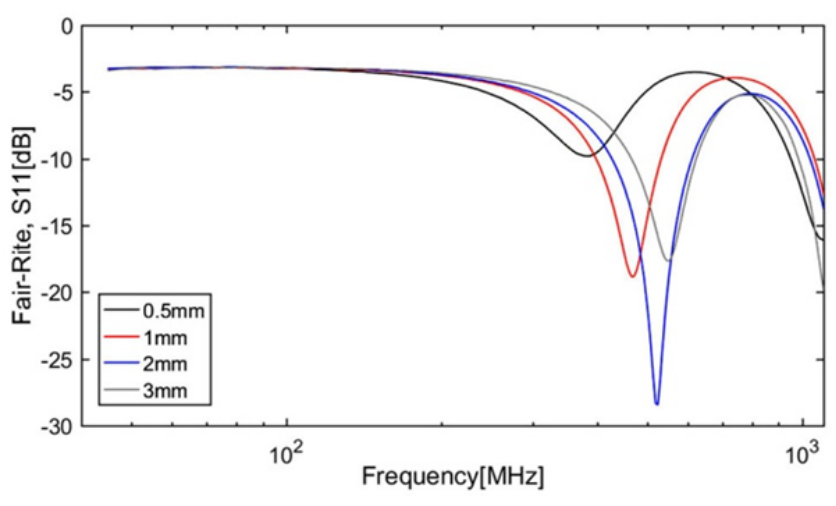

Fig. 7. Measured $S_{11}$ for Fair-Rite prototype for slot distances of $0.5 \mathrm{~mm}$ (black), $1 \mathrm{~mm}$ (red), $2 \mathrm{~mm}$ (blue) and $3 \mathrm{~mm}$ (gray)

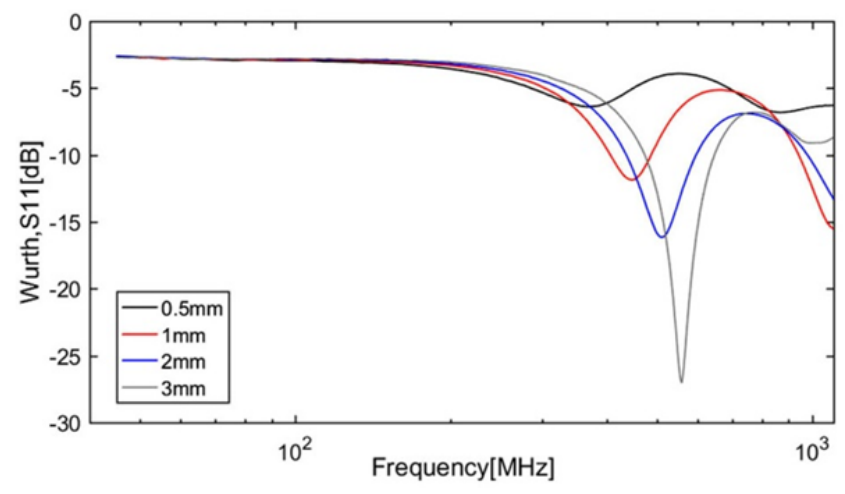

Fig. 8. Measured $S_{11}$ for Wurth prototype for slot distances of $0.5 \mathrm{~mm}$ (black), $1 \mathrm{~mm}$ (red), $2 \mathrm{~mm}$ (blue) and $3 \mathrm{~mm}$ (gray). 


\section{Predicting the Slot Capacitance}

The problem of predicting the slot capacitance can be simplified to the problem of predicting the capacitance between two axially centered cylindrical conductors spaced by a distance $d$ as shown in Fig. 1(b). There is no accurate analytical formula that can help to calculate the capacitance of such geometrical configuration. Therefore, numerical methods using CST-MWS or COMSOL Multiphysics can be used.

To introduce the effect of its permittivity, the core is considered in the model as shown in the cross-sectional view of Fig. 9. The shield is defined as a perfect conductor, and for getting high precision the adaptive mesh refinement choice is checked in the Electrostatic Solver (ES). In Simulation, the permeability spectra of Fig. 5 are used, and the capacitance prediction is carried out for three different constant spectra of relative permittivity $\varepsilon_{\mathrm{r}}=(1,10$ and 100).

Even though the value of relative permittivity for $\mathrm{NiZn}$ ferrites is small at high frequencies, it is intended to know the effect of higher permittivity. Simulation shows that the permittivity of the core doesn't affect the predicted slot capacitance even for high values, this is due to the core relative location in respect to the main plates defining the capacitor, and the relatively big distance between them, therefore the core can be omitted from the model of Fig. 9 while predicting the slot capacitance.

Slot capacitance values as predicted from CST model for the proposed prototypes are listed in Tab. 2. In case of $0.5 \mathrm{~mm}$, the slot capacitance of $4.4 \mathrm{pF}$ has an impedance of $100 \Omega$ at $360 \mathrm{MHz}$, where the minimum of reflection coefficient occurs. In case of $3 \mathrm{~mm}$, the slot capacitance of $1.6 \mathrm{pF}$ has an impedance of $180 \Omega$ at $550 \mathrm{MHz}$, where its minimum of reflection coefficient occurs. These values are still high when compared with the reference impedance of $50 \Omega$, this means that the slot capacitance is not the only capacitor affecting these minimum, as we will see later.

\section{Input Impedance Spectra}

To allocate the frequencies at which resonance occurs, and for better understanding of the base of precedent results, the imaginary part of measured input impedance is investigated. Regarding Fig. 10 for Fair-Rite core prototype and Fig. 11 for Wurth one, the relation between the decrease of slot capacitance and the shift of the curve towards higher frequencies, can be noted.

It can be seen too, that the lower band of measuring rang represents a capacitive region, at which the imaginary part of input impedance is negative, this region is definitely later to the main inductive region at lower frequencies (below the band of measurement). This means that there is a resonance frequency below $45 \mathrm{MHz}$ at which the value of imaginary part of input impedance becomes zero. This resonance frequency in case of Wurth core is higher than that for Fair-Rite in all cases.

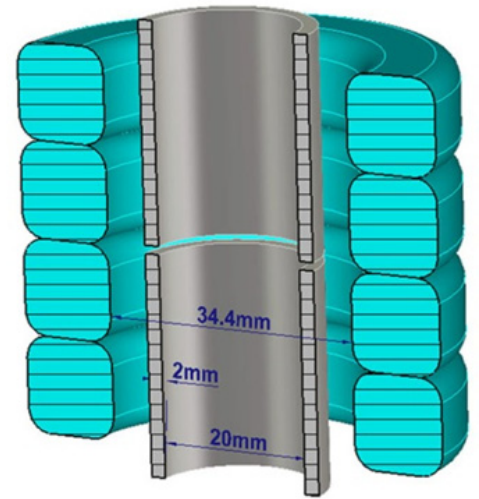

Fig. 9. A cross section of CST-model used to predict the slot capacitance.

\begin{tabular}{|c|c|}
\hline Slot Distance $d$ & Predicted $C_{\mathrm{S}}$ \\
\hline $0.5 \mathrm{~mm}$ & $4.4 \mathrm{pF}$ \\
\hline $1 \mathrm{~mm}$ & $2.9 \mathrm{pF}$ \\
\hline $2 \mathrm{~mm}$ & $1.8 \mathrm{pF}$ \\
\hline $3 \mathrm{~mm}$ & $1.6 \mathrm{pF}$ \\
\hline
\end{tabular}

Tab. 2. Predicted slot capacitance from CST model.

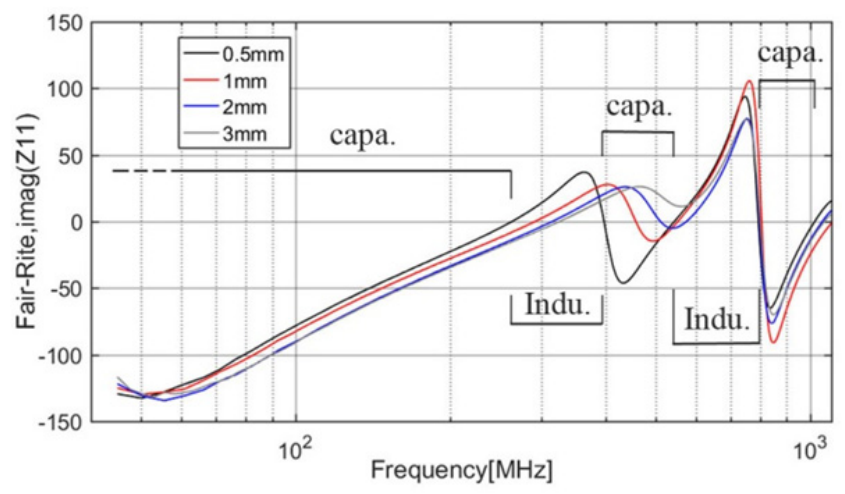

Fig. 10. Measured imaginary part of input impedance for FairRite prototype for slot distances of $0.5 \mathrm{~mm}$ (black), $1 \mathrm{~mm}$ (red), $2 \mathrm{~mm}$ (blue) and $3 \mathrm{~mm}$ (gray). The capacitive (capa.) and inductive (Indu.) regions were set with respect to black curve.

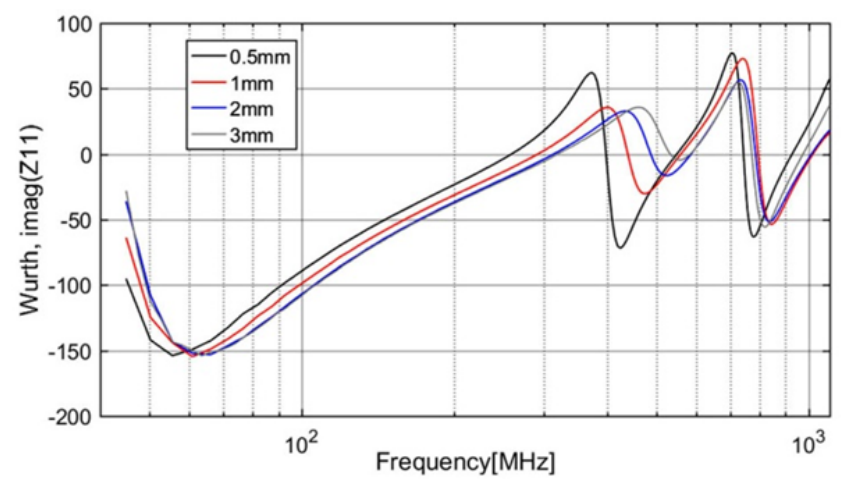

Fig. 11. Measured imaginary part of input impedance for Wurth prototype for slot distances of $0.5 \mathrm{~mm}$ (black), $1 \mathrm{~mm}$ (red), $2 \mathrm{~mm}$ (blue) and $3 \mathrm{~mm}$ (gray).

The lower band capacitive region is followed by another capacitive region later to an inductive region at which the effect of the slot capacitance mostly appears, therefore, 
for distinction, it is called here, the slot capacitive region. When the imaginary part of input impedance of Fig. 10 is compared with its related $S_{11}$ of Fig. 7, it can be concluded that minimums of $S_{11}$ occur at the peaks of said inductive regions. Since these peaks occur at the same frequencies for Wurth core prototype, an inductance other than the material inductance is responsible for it, which is the leakage inductance. Since the two prototypes are identical, they have the same leakage inductance, the parameters contributing the leakage inductance would be studied in detail later using EM model.

As the slot capacitance decreases, the slot capacitive region decreases too, finally, it disappears at a slot distance bigger than $3 \mathrm{~mm}$ and no resonance because of it occurs. After this end the two inductive regions join together, which means that the last two inductive regions have the same source parameters.

After the slot capacitive region, another end-band capacitive region appears in measured input impedance, the slot capacitance shows a little effect in this region. In case of Fair-Rite prototype, the resonance occurs at $800 \mathrm{MHz}$ for all cases, while in case of Wurth prototype, the resonance frequency experiences small changes. To analyze the main source parameters affecting each region, a CST-MWS model for the probe is implemented.

\section{Electromagnetic Model}

A 3D EM model for the proposed BCI-probe, is implemented in CST-MWS, the sectional view of the model is shown in Fig. 12. Aluminum and copper parts are considered as perfect electric conductors, while Teflon properties are downloaded from CST materials library. The text file defining the complex permeability of Fig. 5 for Fair-Rite is used for magnetic dispersion. The relative permittivity of $\mathrm{NiZn}$ ferrite materials doesn't exceed 15 for frequencies above $50 \mathrm{kHz}$ [22], it was found to decrease with the increase in frequency in ferrite which can be considered as a normal dielectric behavior, this decrease is rapid at lower frequencies and slower at higher frequencies [23]. Thus, considering the permittivity constant at the proposed measuring range of frequencies is an acceptable approximation.

To bring to light its effect, a relative dielectric constant of 25 is chosen in the model. Frequency Domain Solver is applied and adaptive mesh refinement choice is checked in the solver to increase the precision.

The result of simulation in Fig. 13 (black curve), shows the same measured inductive and capacitive regions. In the following, the parameters affecting each region would be studied.

To simplify the comparison of results and facilitate their understanding, the following encoding is used: $\mathrm{P}$ - relative permittivity, $\mathrm{W}$ - the width of the turn in $\mathrm{mm}$, $\mathrm{d}$ - the slot distance in mm. Example, P25W10d1: means that the core relative permittivity is 25 , the width of the turn is $10 \mathrm{~mm}$ and the slot distance is $1 \mathrm{~mm}$.

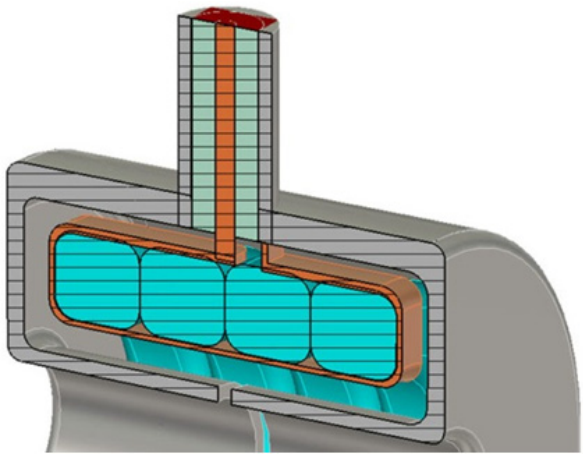

Fig. 12. Sectional view of CST EM-model for the BCI-probe.

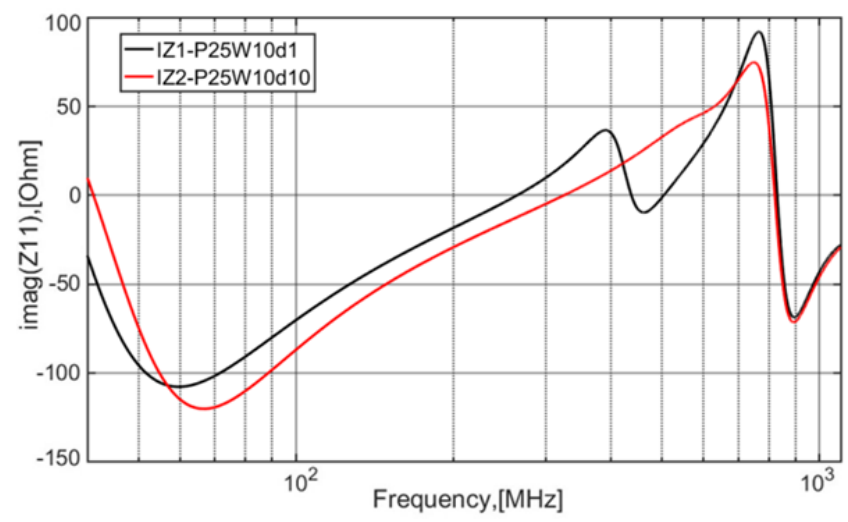

Fig. 13. Imaginary part of predicted input impedance from EMmodel (black), and the result of cancelling the effect of slot capacitance (red)

\subsection{Effect of Slot Capacitance and Leakage Inductance}

To determine the range frequency limits at which the slot capacitance contributes the input impedance, its effect is cancelled by making the slot distance big enough. Thus, in Fig. 13, the result of choosing $d=10 \mathrm{~mm}$ (red curve) shows that the slot capacitance contributes the two first capacitive regions. The shift of the curve towards high frequencies comes from the decrease resultant in the slot capacitance. When the slot capacitance is omitted the second and third inductive regions become combined together as in measurement, which assures that they have the same source parameters.

As far as we know, increasing the copper-covered area of the core decreases the leakage inductance. To test that, the turn width is made $15 \mathrm{~mm}$ instead of $10 \mathrm{~mm}$. The result of comparing predicted input impedance is shown in Fig. 14. It illustrates that, compared to the case of $W=10 \mathrm{~mm}$ (red curve), the curve submits a left shift below about $800 \mathrm{MHz}$ in case of $W=15 \mathrm{~mm}$ (blue curve), which reflects an increase in a capacitive effect, and right shift above $800 \mathrm{MHz}$ which comes from the decrease in leakage inductance.

Actually, the inter windings capacitor, formed between the primary turn and the shield, increases by increasing the width of primary turn, and its effect dominates the leakage inductance effect at low frequencies. Figure 14 


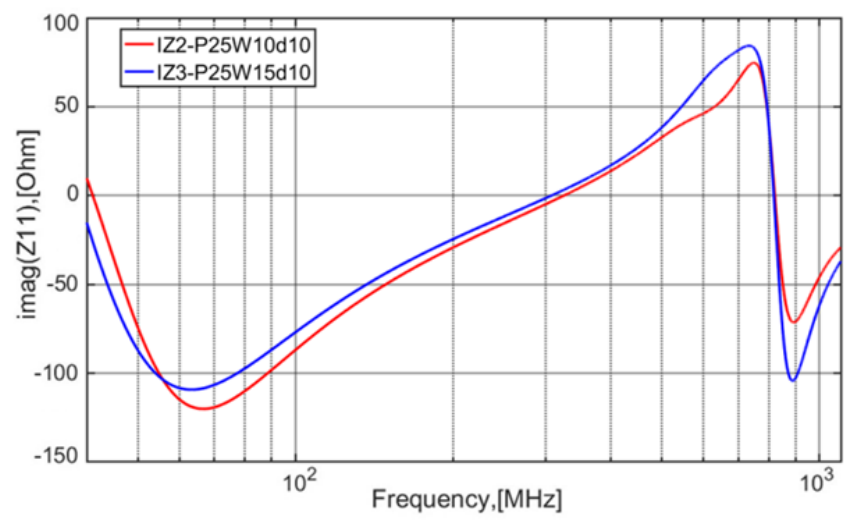

Fig. 14. Effect of making the turn width $15 \mathrm{~mm}$ (blue), instead of $10 \mathrm{~mm}$ (red), on the imaginary part of predicted input impedance.

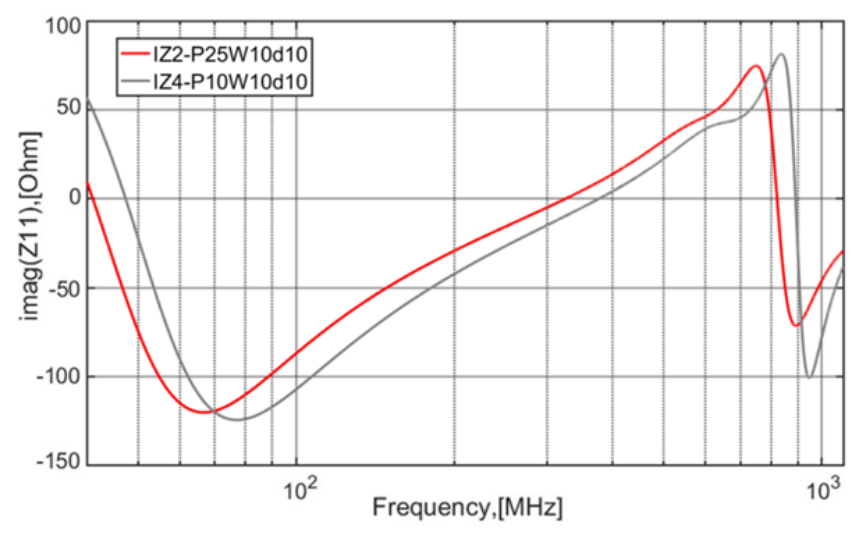

Fig. 15. Effect of the core permittivity on predicted input impedance.

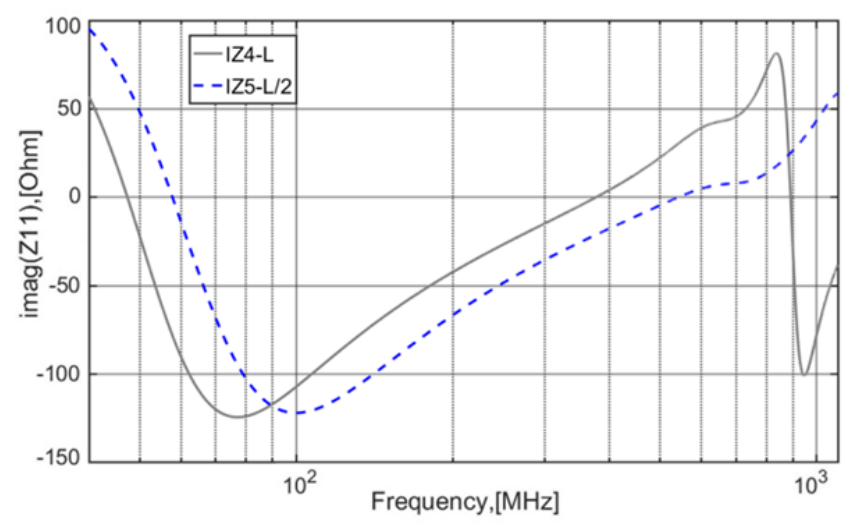

Fig. 16. Effect of decreasing the length $\mathrm{L}$ of the connector to half its basic value.

shows that the effect of inter windings capacitor contributes a wide bandwidth, therefore, it can't be neglected while modeling the BCI-probe.

\subsection{The Capacitive Effect of Ferrite Permittivity}

In Fig. 15, to see its effect, ferrite relative permittivity is decreased from 25 (red curve), to 10 (gray curve). Predicted input impedance indicates that ferrite permittivity is responsible for a capacitive contribution at all the measur- ing bandwidth, this reveals the role of permittivity in magnetic devices as introducing capacitive influence, which results in a resonance phenomenon in the device.

Backing to Figs. 10 and 11, it can be concluded that Fair-Rite core has a higher permittivity than Wurth core. Actually, the weight of Fair-Rite core is much higher than that of Wurth, and since they have the same size, this means that Fair-Rite core has a higher density, this interprets why it has a higher permittivity [24].

\subsection{The Capacitive Effect of the Connector}

To illustrate the capacitive contribution of the connector, its length is made half of its basic one as shown in Fig. 16. Predicted input impedance shows a comparable shift of the curve towards high frequencies at all the proposed band because of the achieved decrease in the connector capacitance. This proves that the connector has a dominant capacitive effect which forms an important factor in creating resonance phenomenon in the probe.

\subsection{Effect of the Shield}

Two capacitive effects were presented for the shield, the slot and inter windings capacitance. In fact, the distance between the primary turn and the shield affects the inter windings capacitance and leakage inductance. To show that, the separating distances between the primary turn and the shield are increased by $1 \mathrm{~mm}$ from all sides, but the inner one, because it would affect the value of slot capacitance.

In Fig. 17, the effect of making the shield wider (dashed-red curve), on the imaginary part of predicted input impedance, is illustrated. The right shift of the curve below $800 \mathrm{MHz}$ reflects the decrease resultant in inter winding capacitance; while the left shift above $800 \mathrm{MHz}$, in the inductive region, reflects the increase in leakage inductance. Thus, decreasing the inter winding capacitance by widening the shield increases the leakage inductance, and decreasing the inter winding capacitance by decreasing the width of the turn increases the leakage inductance and copper losses because of the increase in the coil resistance.

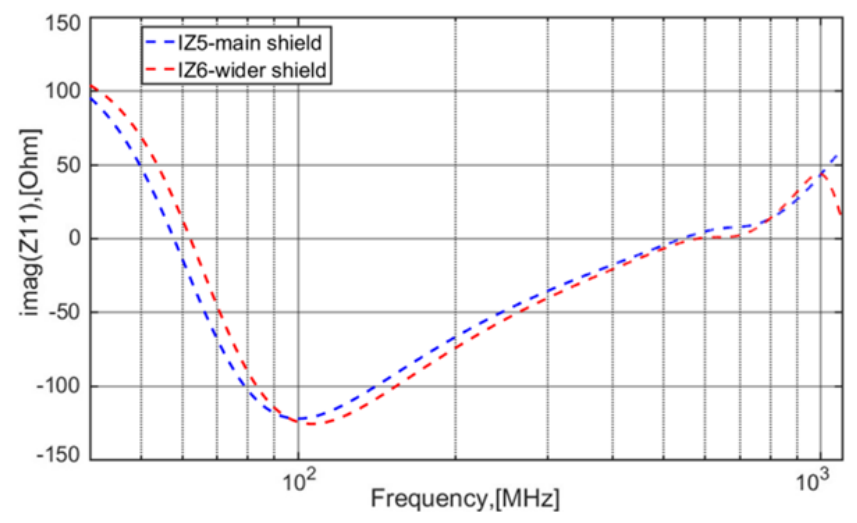

Fig. 17. Effect of increasing the space distances between the shield and the primary turn. 


\section{Validation}

Chamber of the shield of Fair-Rite prototype was altered to widen the space distance between the shield and the core by $1 \mathrm{~mm}$ from each side, except the inner one. The result of measuring the input impedance (blue curve) compared to the previous case (gray curve), is shown in Fig. 18. The same behavior said in the previous section from EM model is observed above and below $800 \mathrm{MHz}$. Apart from the last change, the slot distance was increased to $20 \mathrm{~mm}$, so that its effect can be canceled, the result of achieving the proposed change is shown in Fig. 19 (red curve); compared to the previous distance of $3 \mathrm{~mm}$ (blue curve), the right shift of the curve reflects the decrease in slot capacitance, and the complete merge of the two inductive regions proves practically that they have the same source parameter.

\section{Conclusion}

The effect of the slot capacitance of the shield on its measured quantities has been studied practically by fabricating two identical BCI-probes using two ferrite core materials with the same size and different electromagnetic characteristics. The slot distance of the shield has been changed for the two proposed probes to see its effect on measured $S_{11}$. Measurements indicate that the slot affects

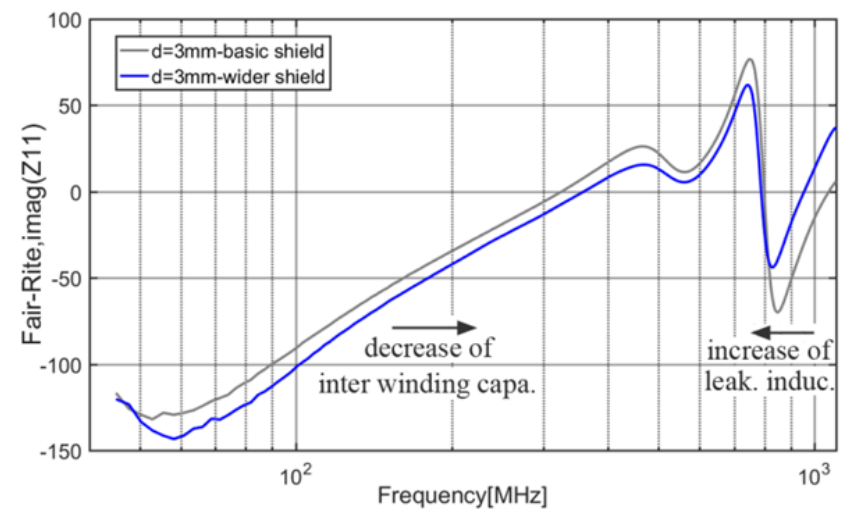

Fig. 18. Practical validation of the effect of widening the distance between the shield and the core by $1 \mathrm{~mm}$.

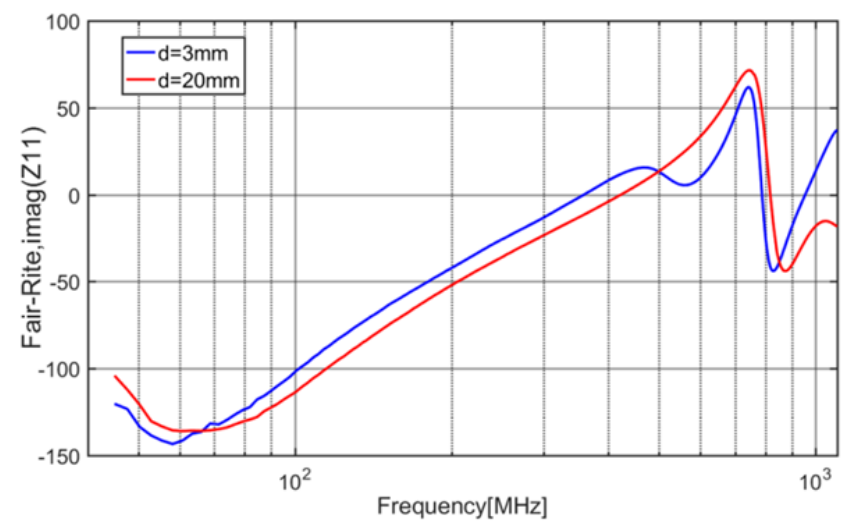

Fig. 19. Practical validation of the effect of eliminating the effect of slot capacitance by increasing the slot distance to $20 \mathrm{~mm}$ similarly for the two materials, and for a specified slot distance, minimums of $S_{11}$ occur at the same frequency for the two prototypes.

The imaginary part of measured impedance shows that the BCI-probe spectra include three capacitive regions, each one is later to an inductive region. The results showed that the second inductive region at which the slot capacitance shows the most effect doesn't relate to the core material.

An electrostatic model was implemented in CSTMWS, to help predicting the value of slot capacitance, it showed that ferrite core doesn't need to be considered in the model. An EM model was implemented in CST-MWS, it shows the same said regions. The analysis on EM model showed that there are four parameters that share the responsibility for the appearance of these capacitive regions: The slot capacitance, inter winding capacitance, connector and ferrite permittivity capacitive effect.

Besides the slot capacitance, the shield can affect the inter winding capacitance and leakage inductance. Increasing the distance between the primary and the shield decreases the inter winding capacitance and increases the leakage inductance, while increasing the turn width decreases the leakage inductance and copper losses, but increases the inter winding capacitance.

\section{References}

[1] RADIO TECHNICAL COMMISSION FOR AERONAUTICS (RTCA). DO-160F. Environmental Conditions and Test Procedures for Airborne Equipment. 2007.

[2] ELECTROMAGNETIC COMPATIBILITY (EMC). Part 4-6: Testing and Measurement Techniques - Immunity to Conducted Disturbances, Induced by Radio-frequency Fields. IEC Standard 61000-4-6, Ed. 4, 2013.

[3] ISO STANDARD 11451-4. Road Vehicles-Vehicle Test Methods for Electrical Disturbances from Narrowband Radiated Electromagnetic Energy-Part 4: Bulk Current Injection (BCI). June 2006.

[4] ISO STANDARD 11452-4. Road Vehicles- Component Test Methods for Electrical Disturbances from Narrowband Radiated Electromagnetic Energy-Part 4. Dec. 2011.

[5] IEC 62132-3, Ed.1. Integrated Circuit Measurements of Electromagnetic Immunity $150 \mathrm{kHz}$ to $1 \mathrm{GHz}$, Part 3: Bulk Current Injection (BCI) Method. 2007.

[6] DEPARTEMENT OF DEFENSE INTERFACE STANDARD. Requirements for the Control of Electromagnetic Interference Characteristics of Subsystems and Equipment. MIL-STD-461E, Aug. 20, 1999.

[7] CERRI, G., DE LEO, R., PRIMIANI, V. M., et al. Wide-band characterization of current probes. IEEE Transactions on Electromagnetic Compatibility, 2003, vol. 45, no. 4, p. 616-625. DOI: 10.1109/TEMC.2003.819061

[8] GRASSI, F., PIGNARI, S. A., MARLIANI, F. Improved lumped$\mathrm{Pi}$ circuit model for bulk current injection probes. In IEEE Symposium on Electromagnetic Compatibility. Chicago (IL, USA), 2005, p. 451-456. DOI: 10.1109/ISEMC.2005.1513557 
[9] GRASSI, F., MARLIANI, F., PIGNARI, S. A. Circuit modeling of injection probes for bulk current injection. IEEE Transactions on Electromagnetic Compatibility, 2007, vol. 49, no. 3, p. 563-576. DOI: 10.1109/TEMC.2007.902385

[10] NAYAK, B. P., DAS, A., VEDICHERLA, S. R., et al. Circuit models for bulk current injection (BCI) clamps with multiple cables. In IEEE International Symposium on Electromagnetic Compatibility (EMC). Singapore, 2018, p. 1160-1163. DOI: 10.1109/ISEMC.2018.8393970

[11] ZHAO, W., YAN, Z., LIU, W. Two methods for BCI probe to improve the high frequency performance. In The $11^{\text {th }}$ International Symposium on Antennas, Propagation and EM Theory (ISAPE). Guilin (China), Oct. 2016, p. 815-819. DOI: 10.1109/ISAPE.2016.7834082

[12] MURANO, K., TAKATA, N., TAYARANI, M., et al. Analysis of transmission line loaded with BCI probe using circuit concept approach. IEICE Communications Express, 2015, vol. 4, no. 7, p. 223-227. DOI: $10.1587 /$ comex.4.223

[13] MURANO, K., KAMI, Y., TAYARANI, M., et al. Theoretical analysis of BCI test system using circuit concept approach. In IEEE International Symposium on Electromagnetic Compatibility (EMC). Ottawa (ON, Canada), 2016, p. 600-603. DOI: 10.1109/ISEMC.2016.7571716

[14] MURANO, K., HOSHINO, M., TAYARANI, M., et al. Modeling of transmission line loaded with BCI probe using circuit concept approach. In IEEE International Symposium on Electromagnetic Compatibility (EMC). Angers (France), 2017, p. 1-4. DOI: 10.1109/EMCEurope.2017.8094697

[15] LAFON, F., BELAKHOUY, Y., DE DARAN, F. Injection probe modeling for bulk current injection test on multi conductor transmission lines. In Proceedings of the IEEE Symposium on Embedded EMC. Rouen (France), 2007.

[16] GRASSI, F., MARLIANI, F., PIGNARI, S. A. SPICE modeling of BCI probes accounting for the frequency-dependent behavior of the ferrite core. In XIXth General Assembly of International Union of Radio Science (URSI). Chicago (IL, USA), 2008, p. 1-4.

[17] DIOP, M. S., CLAVEL, E., CHEAITO, H., et al. 2D modeling of bulk current injection probe and validation with measurements. In The 32nd General Assembly and Scientific Symposium of the International Union of Radio Science (URSI GASS). Montreal (Canada), 2017, p. 1-4. DOI: 10.23919/URSIGASS.2017.8105244

[18] DE ROY, P., PIPER, S. Full-wave modeling of bulk current injection probe coupling to multi-conductor cable bundles. In IEEE International Symposium on Electromagnetic Compatibility. Ottawa (Canada), 2016, p. 770-774. DOI: 10.1109/ISEMC.2016.7571746

[19] TOSCANI, N., GRASSI, F., SPADACINI, G., et al. Circuit and electromagnetic modeling of bulk current injection test setups involving complex wiring harnesses. IEEE Transactions on Electromagnetic Compatibility, 2018, vol. 60, no. 6, p. 1752-1760. DOI: 10.1109/TEMC.2018.2794823

[20] FISCHER CUSTOM COMMUNICATIONS. BCI Brochure.
[21] FAIR-RITE PRODUCTS CORPORATION. FairRite_Catalog_17th_Edition.

[22] RAMA KRISHNA, K., RAVINDER, D., VIJAYA KUMAR, K. et al. Dielectric properties of Ni-Zn ferrites synthesized by citrate gel method. World Journal of Condensed Matter Physics, 2012, vol. 2, no. 2, p. 57-60. DOI: 10.4236/wjcmp.2012.22010

[23] GHODAKE, U. R., KAMBALE, R. C., SURYAVANSHI, S.S. Effect of $\mathrm{Mn}^{2+}$ substitution on structural, electrical transport and dielectric properties of $\mathrm{Mg}-\mathrm{Zn}$ ferrites. Ceramics International, 2017, vol. 43, no. 1, part B, p. 1129-1134. DOI: $10.1016 /$ j.ceramint.2016.10.053

[24] FAHIRUDDIN ESA, ZULKIFLY ABBAS, FADZIDAH MOHD IDRIS, et al. Characterization of $\mathrm{Ni}_{\mathrm{x}} \mathrm{Zn}_{1-\mathrm{x}} \mathrm{Fe}_{2} \mathrm{O}_{4}$ and permittivity of solid material of $\mathrm{NiO}, \mathrm{ZnO}, \mathrm{Fe}_{2} \mathrm{O}_{3}$, and $\mathrm{Ni}_{\mathrm{x}} \mathrm{Zn}_{1-x} \mathrm{Fe}_{2} \mathrm{O}_{4}$ at microwave frequency using open ended coaxial probe. International Journal of Microwave Science and Technology, 2015, vol. 3, p. 1-8. DOI: 10.1155/2015/219195

\section{About the Authors ...}

Issa Moustafa MASHRIKI was born in Masiaf, Syria. In 1999, he received his B.Sc. in High Power Physics from HIAST, Damascus, Syria. In 2009, he received the M.Sc. degree in Communication Engineering from MUT, Tehran, Iran. From 2016, he is working toward the Ph.D. degree in Electromagnetic Compatibility from MUT. His main research interests are radio waves propagation, antennas analysis and measurements, and EMC.

Seyyed Mohammad Javad RAZAVI was born in Kashan, Iran in 1976. He received the B.Sc. degree from Isfahan University of Technology (IUT), M.Sc. degree from Malek Ashtar University of Technology (MUT), Tehran, and $\mathrm{Ph}$.D. degree from Iran University of Science and Technology (IUST), all in Electrical Engineering in 1999, 2002, and 2009, respectively. His research interests include antenna analysis and measurements, and electromagnetic compatibility.

Seyyed Hossein MOHSENI ARMAKI was born in Kashan, Iran. He received the B.Sc. degree in Communication Engineering from KNTU, Tehran, Iran. In 1995, he received the M.Sc. degree in Communication Engineering from the same university. In 2011, he received the Ph.D. degree from Iran University of Science and Technology, Tehran, Iran. His research interests include antenna analysis, antenna measurements and electromagnetics propagation. 